\title{
Intelligent Grid Initiatives in India
}

\author{
Y. Pradeep, S. A. Khaparde, Senior Member, IEEE, and Reji Kumar
}

\begin{abstract}
Indian power sector is growing at an enormous pace. Building and operating such a power system is a challenging problem. The power network which carries the MWs across large distances is analogous to a "muscle system" of human body. Likewise, the entire range of associated monitoring and control network is analogous to a "nervous system". While strengthening the muscles is important in order to fully realize the potential, they also have to be complemented by an intelligent nervous system. This paper focuses on the intelligent grid aspects and the initiatives taken by the National Grid of India towards that direction. The participation of IBM - Indian Institute of Technology, Bombay (IITB) in the Intelligent Utility Network (IUN) research is also mentioned.
\end{abstract}

\section{INTRODUCTION}

D UE to restructuring of power system, the number and the complexity of the functions that are to be performed by power control centres have increased. So as to keep up with the evolving requirements, the notion of supervisory control is being replaced by intelligent distributed control of the system. Unidirectional centrally controlled nature of existing infrastructure can be upgraded into an interactive, electronically enhanced grid that can spot potential problems in real time, and automatically prevent or correct any faults or disturbances [1]. Vendor dependent non standard legacy devices with proprietary software and proprietary communication protocols are not suitable in this changing environment. The power systems today, are far behind in utilizing the powerful features that modern information and communication technologies provide. This paper describes the current situation of the control of Indian power system and identifies opportunities of making the grid intelligent.

The paper is organized in the following way - First a brief description and the projected growth of the Indian Power System is provided. We then look at the hierarchy of control centres, their roles and responsibilities. Going further into the details, the next section explains the control centre architecture known as Unified Load Despatch and Communications (ULDC) scheme. RTUs form the fundamental data collection devices and are the inputs for Supervisory Control and Data Acquisition (SCADA). The practical issues in operating the control centres and their limitations are discussed. The next section lays down the key principles for the future Energy Management System (EMS) Architecture. To achieve this, we introduce the paradigm of Intelligent Utility Network. The components, key characteristics, and the benefits of Intelligent

Y. Pradeep is with Department of Electrical Engineering, Indian Institute of Technology. email: ypradeep@iitb.ac.in

S. A. Khaparde is with Department of Electrical Engineering, Indian Institute of Technology. email: sak@ee.iitb.ac.in

Reji Kumar is Head of Energy and Utilities, IBM India Pvt. Ltd. email: reji.kumar@in.ibm.com grid are explained. Finally, The participation of Indian Institute of Technology, Bombay (IITB) - IBM in the Intelligent Utility Network(IUN) research is mentioned.

\section{BRIEF OUTLINE OF THE INDIAN POWER SYSTEM}

The Indian power system is operated as five Regional grids viz., Northern Regional grid (NR), Western Regional grid (WR), Eastern Regional grid (ER), Southern Regional grid (SR) and North Eastern Regional grid (NER) [2]. Three of the regions, viz. NR, WR and SR suffer from severe deficits while ER is having surplus generation of about 2500 MW and NER is having marginal surplus based on hydro reservoir levels. The regions are connected to each other either through asynchronous links (HVDC back-to-back) or AC links to enable exchange as and when available surpluses. The objectives of interconnecting the five regional grids in the country through synchronous and asynchronous links are to enable transfer of power from surplus regions to deficit regions, to enable optimal development and utilization of coal, gas and hydro resources in the overall interest of the nation and to improve economy, reliability and quality of power supply.

Since the advent of the current century, the focus of planning the generation and the transmission system in the country has shifted from the orientation of regional selfsufficiency to the concept of optimal utilization of resources on an all India basis [3].

The national grid now operating at present has two synchronous grids, viz. NR-WR-ER-NER (Central grid) and SR (Southern grid) as shown in Fig. 1. In 2007, the national grid meets a peak demand of $90 \mathrm{GW}$, with installed capacity of $132 \mathrm{GW}$. Power flowing from ER/NER to NR/WR/SR is of the order of 5,000 to 6,000 MW. Inter regional transmission capacity of $18,400 \mathrm{MW}$ is available. The majority of the inter regional power transfer takes place over $400 \mathrm{kV}$ Network. However, introduction of 765KV EHVAC Lines and 800KV HVDC lines is being planned [4]. Table I shows the projected growth of the National Grid.

\section{THE OPERATIONAL \& CONTROL HIERARCHY OF INDIAN POWER GRID}

In this section we present the operational \& control hierarchy of Indian Power Grid. The control of the grid is planned to be done at 3 levels of hierarchy namely (1) National Load Despatch Centre (NLDC) (2) Regional Load Despatch Centre (RLDC) and (3) State Load Despatch Centre (SLDC). The roles and responsibilities of each of them are 


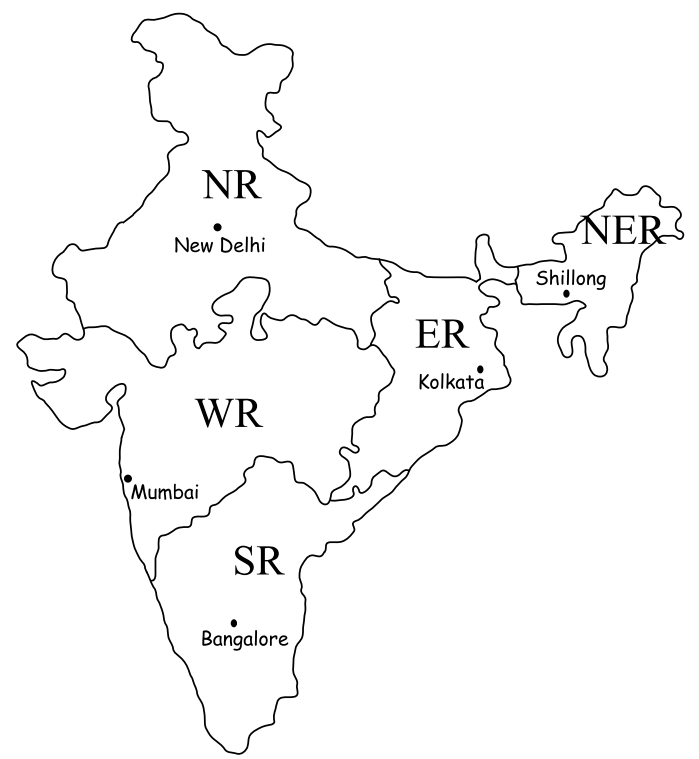

Fig. 1. Regions of Indian Power Grid

TABLE I

PROJECTIONS OF NATIONAL GRID

\begin{tabular}{l|l|l}
\hline National Grid & as on 2007 & by 2012 \\
\hline \hline Installed Capacity & $132 \mathrm{GW}$ & $212 \mathrm{GW}$ \\
\hline Peak Demand & $90 \mathrm{GW}$ & $157 \mathrm{GW}$ \\
\hline $\begin{array}{l}\text { Power from } \\
\text { ER/NER to } \\
\text { NR/WR/SR }\end{array}$ & $6000 \mathrm{MW}$ & $25000 \mathrm{MW}$ \\
\hline $\begin{array}{l}\text { Transmission } \\
\text { Network }\end{array}$ & $\begin{array}{l}400 \mathrm{kV} \mathrm{HVAC} \\
\text { and } 500 \mathrm{kV} \\
\text { HVDC }\end{array}$ & $\begin{array}{l}765 \mathrm{kV} \text { EHVAC and } \\
800 \mathrm{kV} \text { EHVDC }\end{array}$ \\
\hline
\end{tabular}

explained below.

\section{A. National Load Despatch Centre (NLDC)}

The Electricity Act 2003 [5], has provided for constitution of the National Load Despatch Centre for optimum scheduling and despatch of electricity among the RLDCs. As of now the NLDC for India is not yet commissioned for operation. As per policy, it is envisaged that in future all RLDCs shall report to proposed NLDC for Unified Grid Operation at National level. At present the national grid is operated by mutual coordination of RLDCs.

Role of NLDC: The NLDC shall be an apex body to ensure integrated operation of the national power system and discharge the following functions:

- Supervision over the RLDCs.

- Scheduling and despatch of electricity over inter-regional links in accordance with Grid standards specified by the authority and Grid code specified by the Central Commission in coordination with RLDCs.

- Coordination with RLDCs for achieving maximum economy and efficiency in operation of National Grid.

- Monitoring of operations and grid security of the National Grid.
- Supervision and control over the inter regional links as may be required for ensuring stability of the power system under its control.

- Coordination for restoration of synchronous operation of National Grid with RLDCs.

\section{B. Regional Load Despatch Centres (RLDC)}

The Electricity Act 2003, requires the Central Government to make regional demarcation of the country for efficient, economical and integrated transmission and supply of electricity. In particular this demarcation would facilitate voluntarily inter-connection and co-ordination of facilities among the inter-state, regional and inter-regional generation and transmission of electricity. To ensure integrated operation of power system in each such region, the RLDC has been envisaged as an apex body [6], [7], [8], [9], [10]. The directions given by the RLDC for ensuring grid stability etc. are to be complied with by the licensees, generating company, generating stations, sub-stations and any other person connected with the operation of the power system.

Role of RLDC: The RLDC is the apex body to ensure integrated operation of the power system in the Region.

- Monitoring regional grid security parameters in Real time for integrated operation of power system.

- Daily scheduling and operational planning.

- Facilitating interstate / inter regional exchanges.

- Analysis of power system disturbance in the region and facilitating immediate preventive measures.

- Computation of energy despatch and drawal values as per Special Energy Meter (SEM).

- Operational system studies and contingency analysis.

- Augmentation of SCADA, Telemetry and Communication facilities.

- System studies, planning and contingency analysis.

\section{State Load Despatch Centres (SLDC)}

Corresponding to the RLDC which operates at the regional level, the SLDCs have been envisaged at the State level with the responsibility of ensuring integrated operations of the power system in State.

\section{Role of SLDC}

- To be responsible for optimum scheduling and despatch of electricity within a State in accordance with the contracts entered into with the licensees or the generating companies operating in that State.

- Monitor grid operation.

- Keep accounts of the quantity of electricity transmitted through the state grid.

- Exercise supervision and control over the inter-State transmission system.

- Be responsible for carrying out real time operation for grid control and despatch of electricity within the State through secure and economic operation of the State Grid 


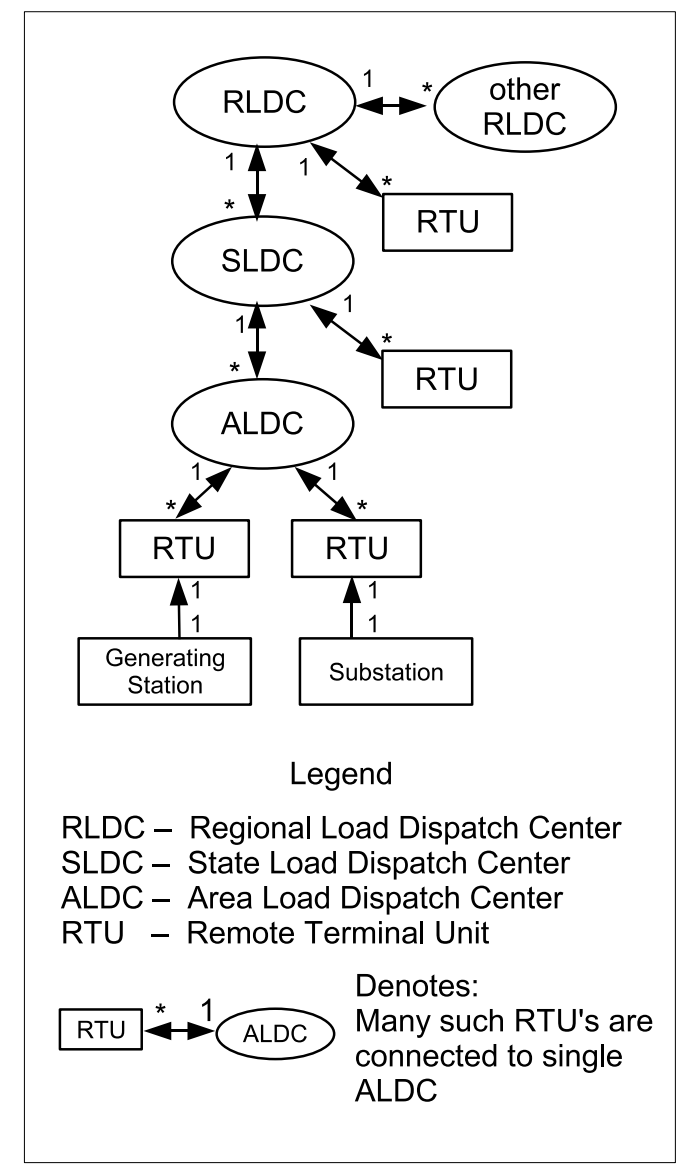

Fig. 2. Control Centre Architecture in India

in accordance with the Grid standards and State Grid Code.

It can be observed that, the control centre infrastructure is arranged in a certain hierarchy, resulting in the need of a well defined scheme for communications among the entities in the hierarchy. Such a scheme known as Unified Load Despatch and Communications Scheme, and its details are presented in the next section.

\section{UNIFIED LOAD DESPATCH AND COMMUNICATIONS (ULDC) SCHEME}

\section{A. Inception}

In May 1992, the Government of India directed all Regional Load Despatch Centres and State Load Despatch Centres, to follow Unified scheme of operation and control. The Unified Load Despatch and Communication (ULDC) project, also referred to as SCandC (System Coordination and Control), has been conceived to monitor, operate and control the regional power grid in a unified and coordinated manner. Monitoring of the grid system based on realtime data is vital for optimal system operation and also to minimize system tripping and blackouts. Besides, the delivery of scheduled power from Central Sector and jointly owned power plants to the beneficiary states requires a hierarchical network of load despatch centres along with adequate telecommunication
TABLE II

RTU'S ACROSS SELECTED REGIONS

\begin{tabular}{l|c}
\hline RLDC & $\begin{array}{c}\text { Number of } \\
\text { reporting RTU's }\end{array}$ \\
\hline \hline NRLDC & 368 \\
NERLDC & 65 \\
SRLDC & 337 \\
WRLDC & 167 \\
\hline
\end{tabular}

facilities.

As a result of the unified approach, we can ensure compatibility of equipment, standardization of hardware and software, well coordinated and phased implementation, optimization of maintenance and spares etc., leading to cost effectiveness.

\section{B. The ULDC control centre architecture}

The ULDC architecture can be thought of as an analogy where, Remote Terminal Units (RTUs) form the eyes and ears of the system, the communication network is the nervous system and the control centre forms the brain for operational and control decisions. The ULDC architecture is shown in Fig. 2. Table II shows the number of RTUs reporting to the RLDCs located in the respective regions in India. Every RTU has a predefined Master Control Centre (CC) and it has to report its data to the CC. An RTU may be connected to an Area Load Despatch Centre (ALDC) or SLDC or RLDC depending on whose data that RTU is reporting. For example, all RTUs connected ISGS (Inter State Generation Stations, owned by Central Government) report directly to RLDC, whereas, RTUs connected to state owned generation are connected to the corresponding SLDC. However, irrespective of the master $\mathrm{CC}$, all RTUs through ALDC-SLDC hierarchy, ultimately report to the RLDC. The communication between RTU and master takes place using IEC protocol and control centres in turn communicate using Inter Control Centre Protocol (ICCP). At hardware level, Fibre Optic Communication Link, Microwave and Power Line Career Communication (PLCC) links are used.

RTUs collect two types of information. First is the measurand information which comprises of continuously varying quantities like MW, MVAR, Amps, Volts, etc. collected from various devices in the substation, stored in measurand file. Second is the status information like isolator position, circuit breaker status, etc. The measurand file is polled by the master CC every 10-20 seconds. But the status information is sent whenever there is a status change, as event based reporting to master by the RTU.

The realtime data, thus collected from the RTUs is fed to the Supervisory Control And Data Acquisition (SCADA) system which aids the system operators in controlling the grid. The direct benefits of a modern SCADA system are [11]:

- Constant access to realtime picture of entire network showing power system voltage, frequency, MW, MVAR, 
etc.

- Supervision, monitoring and control of power in realtime.

- Optimal operation of power system, i.e., generation and associated resources.

- Minimum outage and faster restoration of the system in the event of Grid disturbances.

- Improvement in the quality of supply through better control of frequency, voltage and other parameters.

- Lesser dependence on basic telephone system.

\section{Limitations}

There are some limitations with the current system that need to be addressed.

1) Time Synchronization: The SOE (Sequence of Events) analysis require the recording of the events to be time stamped accurately up to milliseconds. This requires that the control equipment all over the grid must keep track of the time, and that the time must be synchronized with all. Barring some central substations, in most of the substations in India only the RTU is time synchronized with the master CC. All the other equipment in the substation must also be time synchronized.

2) PLCC is Slow: The communication through PLCC, owing to the baud rate of 300 to 600 , is not fast enough. This means that the moment RTUs report a status change by the time it reached to the ALDC it takes some time. However, as the control centres are connected through fibre optics, the communication is faster.

3) Visualization and Navigation: All the data collected by the CC must be monitored, analyzed and decisions must be made. The required data, in the required format, in a required view must be easily available for the system operator. If more number of mouse clicks, menu selection and operations are involved then navigation becomes difficult.

\section{Key principles for EMS/MMS architecture of the future}

The system operators of the very large power grids commonly share some concerns over the requirements of operating the future power system. The following are the key principles for future EMS architecture as explained in [12]

1) Migration to a Component Based, Service Oriented Architecture (SOA), which will promote application re-usability, interoperability and interchangeability of shared components not only within the EMS/MMS environment, but also across the enterprize [13].

2) The adoption of a common data model to enable multivendor implementations, while providing a single consistent source of data within the enterprize.

3) Platform independence to enable the integration of bestof-class third-party applications, regardless of the underlying technologies.

4) Security built as a foundation in the design, not just an after-thought.
5) Business continuity, which addresses both the physical redundancy of the computing environment and the cyber-security concerns.

6) Adoption of emerging industry standards (such as CIM, the Common Information Model), as well as development of standard shareable components and standard validation and certification processes.

7) Wide range scalability, allowing the reuse of common technologies and components across multiple industry segments and industries.

8) Open architecture and published interfaces, allowing the integration of third-party or in-house developed applications.

9) Maintainability, through the ability to upgrade/replace a single component without compromising the rest of the system.

These principles can be realized by the new paradigm of Intelligent Grid, which is discussed in the next section.

\section{INTELligent UtiLity Networks}

This section introduces the Intelligent Utility Network (IUN) and explains its features. IUN is defined as - A power network that makes an extensive use of sensors for grid observability and integrates many data sources to drive analytics, which transform data into useful information, to support advanced operational control and strategic decision making [14].

An intelligent power grid is the one in which we basically add a new network automation layer on top of the existing infrastructure - sensors, communications, software to monitor. This provides the capability to have observability of all the infrastructure at all times.

Intelligent grid consists of five major components i.e. Data sources, Data Transport, Data integration, Analytics and Optimization.

Some key characteristics of intelligent grid are

- Power grid devices are monitored by intelligent devices

- All intelligent devices are IP enabled

- Digital communication networks connect these devices

- Data collection for analysis

- Business intelligence and optimization tools to support advance control and decision support.

Intelligent power grid is a conceptual framework for helping utilities to focus on true business drivers. To move from keep-the-lights-on approach, to reliable delivery of high quality power over stable and secure grid, asset utilization optimization, life cycle management and cost containment.

Intelligent utility network improves reliability, safety, customer satisfaction, response to changing regulations, better workforce utilization and reduced operational cost. The basic approach of intelligent grid is characterized by MonitorAnalyze-Manage as depicted in the Fig. 3. The major block 


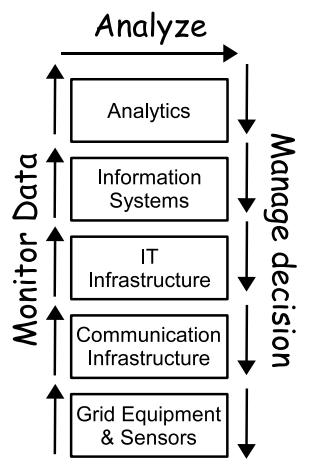

Fig. 3. The approach of Intelligent Grid

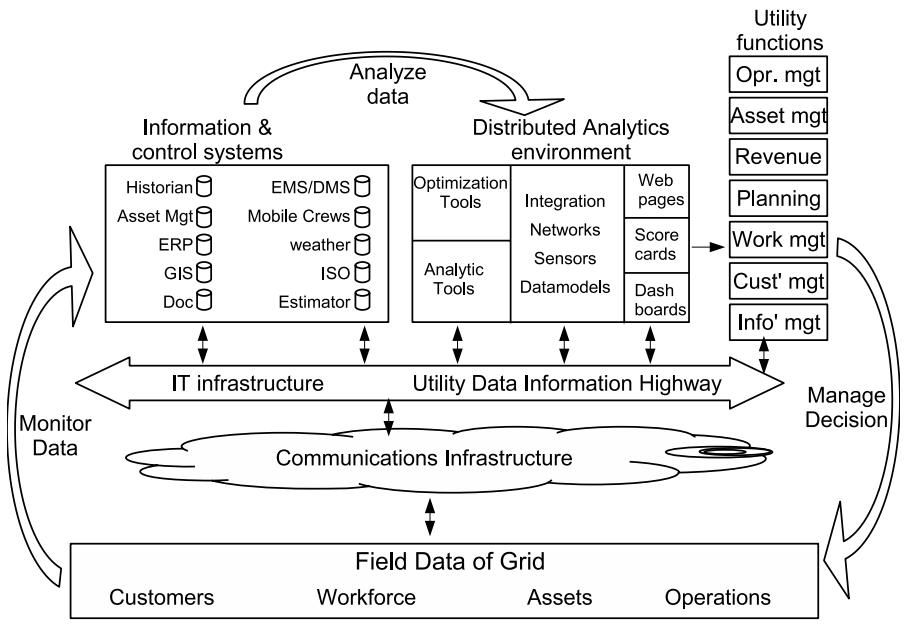

Fig. 4. Major Blocks of Intelligent Utility Network

diagram of Intelligent Utility Network is given in Fig. 4.

A well designed intelligent network will produce a broad range of benefits for utility and its customers [15]. These are elaborated as follows:

1) Asset Lifecycle Management: By real time remote monitoring of the asset health, we can achieve increased asset life, optimize on operation and maintenance cost.

2) Work Management: The duration and frequency of site visits is reduced and at the same time, accurate outage location can be found. This results in proper identification of causes, faster response, prepared, informed and knowledgeable crew.

3) Information Management: The presence of communication information leads to access and sharing of data across various utility applications. There is no redundant or inconsistent data, it is input once and re-used many times. However for all this to happen, standard information models like
CIM (Common Information Model) and IEC 61850 (Substation Automation) must be used.

4) Planning Management: Access to historical operation and asset data improves grid planning, in terms of, accurate design and sizing of new equipment to meet demand growth, investment decisions based on customer profile, optimize capital expenditure or even defer unnecessary capital investments.

5) Grid operation and management: Intelligent devices, sensors and meters eliminate "blind spots". This leads to faster detection and localization of outages, better load balancing, maintaining stability. Better observability of the system can locate issues related to power quality, reliability and fault before they impact customers.

6) Customer Management: IUN enables the operator to meet the customer requirements. It also provides more information to the customers which helps them manage consumption, cost and other decisions like choice of service and price.

7) Revenue Management: An intelligent portal is provided to the consumer, which does the billing. Assurance of revenue is ensured by remote connect/disconnect operations.

\section{The IUN Research PRoJeCt IN INDIA: IITB-IBM PARTICIPATION}

Implementation of Intelligent Utility Network is a very large scale undertaking, involving large investments and many stake holders. The following can be one approach in going about this problem - Developing the IUN strategy, validation of risk, identification and planning, followed by a pilot project, integrate organization and processes and finally continue to roll out in phases.

The significance of intelligent grid has been identified by Power Grid Corporation of India Limited (PGCIL) which is the Central Transmission Utility in India. PGCIL with support of Ministry of Power has taken initiative by launching an international conference "GRIDTECH" in February 2007 [16]. The theme is to identify new technologies in transmission \& distribution and synergic areas like load despatch, telecommunication, application software and to showcase state-of-the-art technologies from reputed manufacturers/vendors.

IBM and IIT Bombay have joined this drive. IBM and IIT Bombay have formed a research cell supported by IBM. IBM is a founding member of Gridwise, USA [17]. The IBMIITB research cell is aimed at identifying the opportunities, implementation issues unique to Indian power scenario and also demonstrating the benefits that an intelligent grid can provide. Since its inception the cell is actively interacting with the utilities and also initiated projects with them. 


\section{A. Project with NRLDC:}

The research cell has initiated a project with Northern Region Load Despatch Center (NRLDC) [6], which is the system operator for the Northern Region of India. The initial step in the project is to develop a Blueprint for Intelligent Power Grid Management, which provides a roadmap to consolidate and integrate the real time as well as the historical data from the SCADA/EMS systems and perform advanced data analytics on the data. In the restructured environment, it is perceived that the existing SCADA/EMS systems are obsolete and as they are proprietary systems, they are restricting the introduction of new advancements. The project aims to enhance the grid operation by addressing the limitations in the existing SCADA/EMS system in terms of operation and control, visualization and decision support.

\section{B. Project with Reliance Energy:}

Reliance Energy is an integrated private sector power utility company which is into generation, transmission, distribution and trading of power [18]. It distributes about $5000 \mathrm{MW}$ of power all over India, and has a state-of-the-art distribution SCADA system. However, the operations are mainly centered around the real time data, whereas, the historical data is mainly used for basic reporting purposes. This project is aimed at developing data mining applications and advanced analytics, for extracting valuable insights about the distribution network operation from the huge database. Various other business scenarios, where such an application can add value are identified as asset lifecycle management, preventive maintenance, efficient grid operation, enhanced grid observability, decision support, etc. As a part of implementation, a data mining application for selected business scenarios was developed as proof-of-concept and demonstrated to the utility to establish the effectiveness. IBM products like DWE, Alphablox and iMiner were used, which provide the basic framework for building such applications.

\section{CONCLUSION}

This paper has given a brief description and the projected growth of the Indian Power System. The hierarchy of control centres, their roles and responsibilities, the control centre architecture are explained. The practical issues in operating the control centres and their limitations are discussed. The key principles for the future EMS Architecture are laid down. The paradigm of Intelligent Utility Network, components, key characteristics, and its benefits are explained.

It is clear that the current day power systems are inadequate to meet the requirements of the future.The notion of supervisory control is being replaced by intelligent distributed control of the system. Intelligent Grid paradigm hold a promise for future and the utilities should embrace it for efficient grid management.

\section{ACKNOWLEDGMENT}

The authors would like to thank Mr. P. Pentayya, Deputy General Manager, Western Region Load Despatch Centre,
Mumbai for his valuable inputs on various practical issues of operating a Regional Load Despatch Centre. The authors also thank Mr. Tata, Deputy General Manager, Northern Region Load Despatch Centre, New Delhi and Dr. Rajamani, Reliance Energy Ltd. Mumbai for their valuable support in the respective projects.

\section{REFERENCES}

[1] J. P. Conti, "Let the grid do the thinking," IET Power Engineer, pp. 34-37, April/May 2006.

[2] A. Roy, S. A. Khaparde, P. Pentayya, S. Usha, and A. R. Abhayankar, "Operating experience of regional interconnections in india," in Proc. IEEE Power Engineering Society General Meeting, pp. 2528-2535, June 2005.

[3] R. G. Yadav, A. Roy, S. A. Khaparde, and P. Pentayya, "India's fastgrowing power sector," IEEE Power \& Energy Magazine, pp. 39-48, July/August 2005

[4] A. K. Asthana, "Theme paper for gridtech 2007," 5th Feb, New Delhi.

[5] "[online] electricity act 2003 available: http://powermin.nic.in/acts _notification/electricity_act2003/preliminary.htm."

[6] "[online] northen region load despatch center. available: www.nrldc.org."

[7] "[online] north eastern region load despatch center. available: www.nerldc.org."

[8] "[online] eastern region load despatch center. available: www.erldc.org."

[9] "[online] westren region load despatch center. available: www.wrldc.com."

[10] "[online] southern region load despatch center. available: www.srldc.org."

[11] "[online] state load despatch center, delhi. available: http://www.delhisldc.org/redirect.aspx?loc=1501."

[12] J. Schinski and A. Steven, "Ems architectures for the 21st century, a collaborative white paper," Very Large Power Grid Operators Conference, Oct 2005.

[13] F. F. Wu, K. Moslehi, and A. Bose, "Power system control centers: past, present and future," Proceedings of the IEEE, vol. 3, no. 11, pp. 1890-1908, Nov 2005.

[14] J. D. Taft, "Blue print for intelligent power grid in the electric utility of the future," IBM Application Innovation Services, Jan 2006.

[15] G. Bartels and M. Hauske, "Ibm's intelligent utility network - an overview," gridtech2007, New Delhi.

[16] "[online] gridtech 2007. available: http://www.powergridindia.com/ pgnew/applications/gridtech2007/10-0001-000.aspx."

[17] "[online] gridwise. available: http://www.gridwise.com/."

[18] "[online] reliance energy ltd. 2007. available: http://www.rel.co.in/welcome.asp."

Y. Pradeep Kumar is currently working towards Ph.D. degree in Electrical Engineering Department at IIT Bombay, India. His research interests include IT application in power systems and power systems restructuring issues.

Shrikrishna A. Khaparde (M'87-SM'91) is a Professor, Department of Electrical Engineering, Indian Institute of Technology Bombay, India. He is a member of the Advisory Committee of Maharashtra Electricity Regulatory Commission (MERC). He is on the editorial board of International Journal of Emerging Electric Power Systems (IJEEPS). He has co-authored books titled, "Computational Methods for Large Sparse Power System Analysis: An Object Oriented Approach," and, "Transformer Engineering: Design \& Practice," published by Kluwer Academic Publishers and Marcel Dekker, respectively. His research area includes distributed generation and power system restructuring.

Reji Kumar is the Head of Energy and Utilities, IBM India Pvt. Ltd. He has diverse international experience of over 25 years in electricity sector. 